\section{A THRESHOLD OF B CELL COSTIMULATORY SIGNALS IS REQUIRED FOR SPONTANEOUS GERMINAL CENTER FORMATION IN AUTOIMMUNITY}

${ }^{1}$ Kristy Chiang, 'Andrea D Largent, ${ }^{1}$ Tanvi Arkatkar, ${ }^{1}$ Christopher D Thouvenel, ${ }^{1}$ Samuel W Du, ${ }^{1}$ Natali Shumlak, ${ }^{1} J o n a t h a n$ Woods, ${ }^{2}$ Quan-Zhen Li, ${ }^{1,3,4}$ David J Rawlings, ${ }^{1,4}$ Shaun W Jackson*. 'Seattle Children's Research Institute, Seattle, WA; ${ }^{2}$ Department of Immunology, University of Texas Southwestern Medical Center, Dallas, TX; ${ }^{3}$ Department of Immunology; ${ }^{4}$ Department of Pediatrics, University of Washington School of Medicine

10.1136/lupus-2021-lupus21century. 104

Background Systemic lupus erythematosus (SLE) is a multisystem autoimmune disease characterized by polyclonal B cell activation. Rather than being downstream targets of $\mathrm{CD}^{+}{ }^{+} \mathrm{T}$ cell activation, $\mathrm{B}$ cells can initiate breaks in $\mathrm{T}$ cell tolerance by orchestrating the formation of spontaneous germinal centers (GC). Engagement of CD28 on $\mathrm{CD}^{+}{ }^{+} \mathrm{T}$ cells with CD80/ CD86 (B7.1/B7.2) on antigen presenting cells (APCs) is required for $\mathrm{GC}$ formation, but recent data suggest a limited role for B cell-intrinsic CD80/CD86 in this process (Watanabe, J Exp Med, 2017). However, whether B cell costimulatory signals are similarly redundant in immunization models vs. humoral autoimmunity is unclear, given differences in (auto) antigen abundance, affinity and adjuvant load.

Methods To determine whether B cell costimulatory signals modulate autoimmune GCs, we used a chimeric model of B cell-driven autoimmunity to contrast the impact of global CD28 and B cell-intrinsic CD80/CD86 deletion in humoral autoimmunity.
Results Whereas myeloid signals were critical for initial CD4 ${ }^{+}$ $\mathrm{T}$ cell priming and CXCR5 upregulation, complete $\mathrm{T}$ follicular helper (Tfh) cell maturation required B cell-intrinsic CD80/ CD86 expression. Surprisingly, loss of CD28 and B cell-intrinsic CD80/CD86 similarly abrogated the formation of spontaneous autoimmune GCs. Interestingly, absent GCs differentially impacted serum autoantibody (autoAb) titers. In keeping with distinct extra-follicular (EF) and GC activation pathways driving lupus autoAb, lack of GCs correlated with loss of RNAassociated autoAb but preserved anti-dsDNA and connective tissue antigen reactivity. These data suggest a prominent role for GC-independent B cell activation via an EF pathway in the genesis of diverse pathogenic autoAb in SLE. Finally, based on CTLA-4 haploinsufficiency promoting spontaneous humoral autoimmunity in humans, we tested whether modulating B cell CD80/CD86 levels impacts spontaneous GCs. Strikingly, heterozygous B cell CD80/CD86 deletion recapitulated the phenotype of complete deletion, resulting in lack of Tfh expansion, GC formation and generation of RNA-associated autoAb.

Conclusion Our data show that during initial interactions between antigen-primed, autoreactive $\mathrm{T}$ and $\mathrm{B}$ cells, a threshold of $\mathrm{B}$ cell costimulatory signals is required for $\mathrm{T}$ cell activation and spontaneous GC formation. In addition, our findings support a model in which both EF and GC activation pathways provide distinct contributions to the lupus autoAb repertoire. Loss of B cell costimulatory signals uniquely dissociates these events, by abrogating the formation of autoimmune GCs without preventing $\mathrm{T}$ cell-dependent EF B cell activation. 